\title{
Trial Summary Parameter
}

National Cancer Institute

\section{Source}

National Cancer Institute. Trial Summary Parameter. NCI Thesaurus. Code C49691.

Individual characteristics of a clinical trial, e.g. description of trial design, trial blinding schema, and primary objective of trial. 